Category: Mental health

Study type: Cohort study

Title: The impact of spousal bereavement for carers of people with dementia

Citation: Shah SM, Carey IM, Harris T, et al. The mental health and mortality impact of death of a partner with dementia. International Journal of Geriatric Psychiatry. 2016; 31:92937.

\title{
Commentary
}

\section{Implications for practice and research}

- This paper improves our understanding of spousal bereavement in dementia and highlights the need for both pre and post bereavement support for family carers.

- It provides good quality evidence that in the year preceding bereavement, spousal carers of people with dementia are at increased risk of psychological distress.

- This research highlights the need for better support for family carers of people with dementia, and addressing the importance of continuity of services.

- Future research should identify both barriers and facilitators of accessing palliative care services and test the efficacy of interventions that aim to improve palliative care for people with dementia and their families.

\section{Context}

Caring for a partner with physical or mental disability and partner bereavement are wellestablished independent risk factors for psychiatric morbidity. Carers of people with dementia are known to be at higher risk of experiencing poor mental health outcomes such as anxiety and depression. Adverse health effects after bereavement on the other hand have been suggested to be absent, due to 'pre-death grief' associated with caring for a partner with dementia. Palliative care is often not offered or accessible to people with dementia and their families. This means both patients and carers are likely to miss out on valuable support. Shah and colleagues explore whether the effects on mental and physical health in the year before and after bereavement differs between those caring for a partner with dementia and carers experiencing other types of bereavement.

\section{Methods}

Using a primary care database, demographically representative of the UK, a total of 2624 older individuals whose partner had died with dementia were matched with 7512 individuals experiencing bereavement of a partner without dementia. Outcomes included whether or not carers had received a diagnosis of depression or were prescribed any psychotropic medication in the year before bereavement, and whether the deceased partner was identified as needing palliative care. Authors also collected data on engagement with primary care services, mortality rates and how many people of those not previously receiving psychotropic medication, did so in the year after bereavement.

\section{Findings}

Older people living with a partner with dementia were more likely to receive a diagnosis of depression and more likely to be prescribed psychotropic medication in the year before bereavement compared to the comparison sample. People with dementia were less likely to receive palliative care compared to patients without a diagnosis of dementia. Adverse health effects in dementia carers seemed to be attenuated in the year after dementia bereavement. Contact with primary care services increased in both groups after loss of partner, and mortality was lower in men experiencing dementia bereavement. The year after 
bereavement, being prescribed psychotropic medication for the first time was lower in those that had experienced dementia bereavement compared to those experiencing other types of bereavement (e.g. cancer, cardiac disease, other chronic illness).

\section{Commentary}

This study is characterised by many methodological strengths including the large sample size, a UK representative sample and a rigidly matched control group. It provides valuable insight into the complexity of the health effects of experiencing bereavement in dementia. The findings of increased risk of psychiatric morbidity in dementia carers prior to the loss of their partner and attenuated health effects after bereavement support the concept of anticipatory grief (Rando, 2000). The results of the study are in line with evidence that anticipatory grief in family carers of people with dementia, is greatest in the moderate to severe stages of dementia and in spousal carers (Chan et al, 2013).

This study has important clinical implications, as it establishes that caring for and bereavement of a partner with dementia is a significant risk factor for mental health distress. Findings of increased risk of depression in dementia carers prior to bereavement suggest that offering psychological interventions and identifying carers at risk should be part of services available. The study also found that people with dementia were less likely to receive palliative care compared to people with cancer or other chronic illness. Provision of psychosocial support is key in improving outcomes in palliative dementia care (Singer et al., 2016), and it is concerning that people with dementia and their families are not accessing this type of support.

This work highlights the need to improve identification of family carers of people with dementia most likely to be at risk of distress associated with bereavement, develop suitable preventive interventions and understand the barriers for accessing palliative care services. Shah and colleagues' research highlights the need for continuity of support, which should be an essential part of dementia care.

\section{References}

1) Rando, T. (2000). Clinical Dimensions of Anticipatory Mourning: Theory and Practice in Working with the Dying, Their Loved Ones, and Their Caregivers. Research Press: Champaign, IL.

2) Chan, D., Livingston, G., Jones, L., Sampson, E.L. (2013). Grief reactions in dementia carers: a systematic review. International Journal of Geriatric Psychiatry, 28: 1-17.

3) Singer, A.E., Goebel, J.R., Kim, Y.S., Dy, S.M., Ahluwalia, S.C., Clifford, M., Dzeng, E., O'Hanlon, C.E., Motala, A., Walling, A.M., Goldberg, J., Meeker, D., Ochotorena, C., Shanman, R., \& Cui, M., Lorenz, K.A. (2016). Populations and Interventions for Palliative and End-of-Life Care: A Systematic Review. Journal of Palliative Medicine. 19(9):995-1008.

\section{Competing interests: None}

\section{Authors:}

Name: Janina Brede

Affiliation: University College London, Division of Psychiatry, London, UK 
Correspondence: University College London, Division of Psychiatry, 149 Tottenham Court Road, London W1T 7NF UK

Email: Janina.brede.14@ucl.ac.uk

and

Name: Vasiliki Orgeta

Affiliation: University College London, Division of Psychiatry, London, UK 